\title{
Why review Reviews
}

\author{
Making a good review article is a team effort, involving authors, editors and referees. In honour \\ of Peer Review Week, happening this month, we spotlight the essential role of peer reviewers.
}

Peer review is a celebrated part of the scientific process quite literally, in the form of Peer Review Week, which occurs each year in September. At the same time, there are calls within the scientific community to reform or even abolish formal peer review, with its critics pointing to costs such as referees' time and effort, and raising questions about what value it adds to the publication process. Indeed, in physics, there exists a long-established and thriving ecosystem of preprints, which are not peer reviewed, as discussed in a Comment in this issue by Paul Ginsparg, founder of arXiv. At Nature Reviews Physics, we require peer review for all our Review articles, which at first may seem unnecessary, given that they are written by expert authors and discuss existing literature rather than new results. So why do we champion peer review in our journal?

One important reason is the range of expertise that the process brings in. As editors, we have deep knowledge of our own areas of physics, but not in all areas we cover in our journal. Referees can spot technical errors but, more importantly, they can help us to gauge whether an article is opinionated but balanced, or simply biased. For articles that aim to be interdisciplinary, referees from different subject areas can give us an idea of how the article might be received in their different fields. We also try to ensure that articles written by experimentalists are seen by theorists, and vice versa, to ensure the final published article is useful for a broad audience.

Besides technical expertise, referees draw on their own experiences as scientists, which depend on all kinds of factors, including career stage, geographical location, gender, institutional environment and so on. Although any referee can and should point out when an article overlooks research from part of the community - and it should not be the job of minoritized scientists to always have to be the ones doing so - it is often the case that referees with different personal and professional backgrounds will spot different oversights. For example, for articles written by European authors, referees from Asia may be able to suggest work from that continent that should be engaged with.

However, even if peer review has benefits, that does not mean that it cannot be refined. The present-day model of peer review as three anonymous reports (the second of which is invariably grumpy) is not the only way science has been reviewed before publication. As historian of science Melinda Baldwin points out in a World View in this issue, peer review has taken many forms in its history - including, in its earliest days, reports that were for editors, but not for authors, and reports that were published alongside articles as separate commentary articles. These days, journals are experimenting with new approaches, including post-publication review.

These different approaches are based on the evolving needs of the scientific community in different times and places. How can we at Nature Reviews Physics evolve to keep up with the physics community we serve? One new direction we are taking is to formally involve early-career researchers (ECRs) in peer review. In this initiative, referees who are more established in their careers are invited to officially involve a $\mathrm{PhD}$ student or postdoc in writing a report. We hope this process will provide training opportunities for ECRs and, because referees can be named in our systems and on our papers, give them recognition for the work they do. We also hope that it will give ECRs the chance to contribute to making our articles more useful for their sector of the community, which is especially important given that ECRs make up a large part of our readership.

We believe the many benefits of peer review make it a worthwhile process that improves each Review article we publish. We are grateful to all our referees for their time and effort - the journal would not be the same without you. 